\title{
THE SEMILATTICES WITH DISTINGUISHED ENDOMORPHISMS WHICH ARE EQUATIONALLY COMPACT
}

\author{
SYDNEY BULMAN-FLEMING, ISIDORE FLEISCHER AND KLAUS KEIMEL
}

\begin{abstract}
We consider universal algebras $(S ;\{\wedge\} \cup E)$ in which $E$ is a set of endomorphisms of the semilattice $(S ; \wedge)$. It is proved in this paper that such an algebra is equationally compact iff (i) every nonempty subset of $S$ has an infimum, (ii) every up-directed subset of $S$ has a supremum, (iii) for every $s \in S$ and every up-directed family $\left(d_{i}\right)$ in $S$ the equality $s \wedge \bigvee d_{i}=$ $\bigvee s \wedge d_{i}$ holds, (iv) for each $f \in E, f\left(\bigwedge s_{i}\right)=\bigwedge f\left(s_{i}\right)$ holds for every family $\left(s_{i}\right)$ in $S$, and (v) for each $f \in E, f\left(\bigvee d_{i}\right)=\bigvee f\left(d_{i}\right)$ holds for every up-directed family $\left(d_{i}\right)$ in $S$. In addition, it is shown that every equationally compact algebra of this type is a retract (algebraic) of a compact, Hausdorff, 0-dimensional topological one. These results reduce to known ones for semilattices without additional structure.
\end{abstract}

By a semilattice with distinguished endomorphisms (abbreviated here to SENDO) we shall mean a universal algebra $(S ;\{\wedge\} \cup E)$ where $\wedge$ is a binary operation on $S, E$ is a set of unary operations on $S$, and in which the following laws hold:

$$
\begin{aligned}
x \wedge y & =y \wedge x, \\
x \wedge(y \wedge z) & =(x \wedge y) \wedge z, \\
x \wedge x & =x, \\
f(x \wedge y) & =f(x) \wedge f(y) \text { for each } f \in E .
\end{aligned}
$$

Thus, a SENDO is a semilattice together with an additional set of unary fundamental operations selected from the endomorphisms of that semilattice.

A universal algebra $(A ; F)$ is called equationally compact if every system of equations with constants in $A$ (i.e. equalities between pairs of terms built up from variables and elements of $A$ using the operations in $F$-see [4] for more details), every finite subsystem of which has a solution in $A$, has a common solution in $A$. Among the previous results on equationally compact semilattices are the following:

(1) (Grätzer and Lakser [5]). A semilattice $(S ; \wedge)$ is equationally compact iff every nonempty subset of $S$ has an infimum, every up-directed subset of $S$ has a supremum, and the equality $s \wedge \bigvee d_{i}=\bigvee s \wedge d_{i}$ holds for all $s \in S$ and all up-directed families $\left(d_{i}\right)$ in $S$.

(2) (Bulman-Fleming [1]). Every equationally compact semilattice is a

Received by the editors October 21, 1976 and, in revised form, April 12, 1978. AMS (MOS) subject classifications (1970). Primary 06A20; Secondary 06A25, 08A25. Key words and phrases. Semilattice, equationally compact, topological semilattice. 
retract (in the algebraic sense) of a compact, Hausdorff, 0-dimensional topological semilattice.

The review article of Taylor [7] provides a valuable guide to the literature on equational compactness, and should be consulted for further background material.

The following theorem states that results (1) and (2) above hold for every SENDO. It contains both these results as special cases (taking $E$ to be empty), and is being used by the first two authors to pursue similar questions in richer ordered structures (e.g. pseudocomplemented semilattices, taking $E$ to be $\left\{{ }^{* *}\right\}$; see [3]).

TheOrem. Let $(S ;\{\wedge\} \cup E)$ be a SENDO. Then the following conditions are mutually equivalent:

(i) $(S ;\{\wedge\} \cup E)$ is equationally compact.

(ii) (a) $(S ; \wedge)$ is equationally compact as a semilattice (see the description (1) above); and in addition

(b) for all families $\left(s_{i}\right)$ in $S$, all up-directed families $\left(d_{i}\right)$ in $S$, and for all $f \in E$ the equalities

$$
f\left(\bigwedge s_{i}\right)=\bigwedge f\left(s_{i}\right) \text { and } f\left(\bigvee d_{i}\right)=\bigvee f\left(d_{i}\right)
$$

hold. (Both sides of each equality exist, by (a).)

(iii) $(S ;\{\wedge\} \cup E)$ is a retract (in the algebraic sense) of a compact, Hausdorff, 0-dimensional topological SENDO.

Proof. (iii) $\rightarrow$ (i). That every retract of a compact, Hausdorff topological (and hence equationally compact) algebra is equationally compact was noted by Mycielski [6].

(i) $\rightarrow$ (ii). (a) follows since the semilattice terms, and hence equations, occur among the SENDO ones. To verify the first equalities of (b), observe that for each $f \in E$ the system

$$
\left\{\wedge f\left(s_{i}\right) \leqslant f(x)\right\} \cup\left\{x \leqslant s_{i}\right\}
$$

(which is of course equivalent to a system of SENDO equations with constants in $S$ ) is finitely solvable in $S$ (by the meet of the occurring $s_{i}$ 's), and thus by (i) is solvable in $S$. The existence of a common solution $c$ yields

$$
\bigwedge f\left(s_{i}\right) \leqslant f(c) \leqslant f\left(\wedge s_{i}\right)
$$

the opposite inequality follows from the order-preserving character of $f$. The second equalities of (b) are proved dually, via the systems

$$
\left\{d_{i} \leqslant x\right\} \cup\left\{f(x) \leqslant \bigvee f\left(d_{i}\right)\right\} \text {. }
$$

(ii) $\rightarrow$ (iii). By an ideal of a conditionally complete $\wedge$-semilattice (such as the semilattice of (ii)) we mean a nonempty initial segment $J$ of $S$, every pair of whose elements has a common upper bound in $S$, with the smallest such being contained in $J$. The set $I(S)$ of all ideals of $(S ; \wedge)$ is closed under arbitrary intersection, and hence $(I(S) ; \cap)$ is a (complete) subsemilattice of 
$\left(2^{S} ; \cap\right)$ into which $(S ; \wedge)$ is (completely) semilattice embedded via $s \mapsto(s]$, the principal ideal determined by $s \in S$. For $J \in I(S)$ and $f \in E$ we define $\bar{f}(J)$ to be the set $\{s \in S \mid s \leqslant f(j)$ for some $j \in J\} . \bar{f}(J)$ is clearly an initial segment of $S$; and if $x$ and $y$ belong to $\bar{f}(J)$, then $x \leqslant f(j)$ and $y \leqslant f(k)$ for certain elements $j, k \in J$. Since $j \vee k$ exists in $J$ and because $f(j \vee k)$ is a common upper bound of $f(j)$ and $f(k), x \vee y$ exists in $S$ and belongs to $\bar{f}(J)$. Thus, $\bar{f}(J) \in I(S)$, and it is easily shown that $\bar{f}$ is an endomorphism of $(I(S)$; $\cap)$ for each $f \in E$. Hence, $(I(S) ;\{\cap\} \cup\{\bar{f} \mid f \in E\})$ is a SENDO, and one can verify that the principal ideal map defined earlier is a SENDO embedding. Furthermore, the map $J \mapsto \bigvee J$ from $I(S)$ to $S$ is a SENDO retraction of this embedding; indeed,

$$
\bigvee J \wedge \bigvee K=\bigvee\{j \wedge k \mid j \in J, k \in K\}=\bigvee J \cap K
$$

for any $J, K \in I(S)$, using the meet-continuity condition of (1), and

$$
f(\bigvee J)=\bigvee f(J)=\bigvee \bar{f}(J)
$$

for any $J \in I(S)$, by the join condition in (ii)(b).

It remains only to prove that $(I(S) ;\{\cap\} \cup\{\bar{f} \mid f \in E\})$ is a compact, Hausdorff, 0-dimensional SENDO. To this end, recall that a subbasis for the product topology on $2^{S}$ (2 with the discrete topology) consists of sets of the form $\left\{T \in 2^{S} \mid s \in T\right\}$, for fixed $s \in S$, and their complements, and that, with respect to this topology, $\left(2^{S} ; \cap\right)$ is a compact, Hausdorff, 0-dimensional topological semilattice. The same is true of $(I(S) ; \cap)$ with respect to the induced topology, for $I(S)$ is closed in $2^{S}$. Indeed, a $T \in 2^{S}$ is not an element of $I(S)$ only if either for some $a \leqslant b \in S$ we have $b \in T$ and $a \notin T$; or, for some $a, b \in T, a \bigvee b$ exists and $a \bigvee b \notin T$; or, for some $a, b \in T, a \bigvee b$ fails to exist in $S$. An open neighborhood of $T$ disjoint from $I(S)$ is obtained in each of these cases by choosing, respectively, $\left\{R \in 2^{S} \mid b \in R, a \notin R\right\}$, $\left\{R \in 2^{S} \mid a, b \in R, a \vee b \notin R\right\}$, and $\left\{R \in 2^{S} \mid a, b \in R\right\}$. Finally, we show that, for each $f \in E, \bar{f}$ is continuous; i.e. that $\bar{f}^{-1}\left(N_{s}\right)$ and $\bar{f}^{-1}\left(D_{s}\right)$ are open for each $s \in S$, where $N_{s}=\{J \in I(S) \mid s \in J\}$, and $D_{s}=I(S)-N_{s}$. If $\{j \in$ $S \mid s \leqslant f(j)\}=\varnothing$, then $\bar{f}^{-1}\left(N_{s}\right)=\varnothing$ and $\bar{f}^{-1}\left(D_{s}\right)=I(S)$ are both open. If $\{j \in S \mid s \leqslant f(j)\} \neq \varnothing$, let $d=\bigwedge\{j \in S \mid s \leqslant f(j)\}$; then since $s \in \bar{f}(J)$ iff $d \in J$ (by the meet condition in (ii)(b)), it follows that $\bar{f}^{-1}\left(N_{s}\right)=N_{d}$ and $\bar{f}^{-1}\left(D_{s}\right)=D_{d}$, which are again both open.

Notes. (1) $I(S)$ in the above theorem is none other than the closure of the image of $S$ in $2^{S}$, so the present construction affords an alternative view to that in [1].

(2) Calling an algebra 1-variable compact if it satisfies the criterion for equational compactness given above restricted to systems of equations involving one and the same variable (say $x$ ), we see from examination of the above proof that the equationally compact SENDOs are exactly the 1-variable compact ones.

(3) The authors are indebted to the referee for pointing out that their 
original proof (dealing only with the case of a single distinguished endomorphism) applies equally well to the present theorem, and also that this theorem has the corollary that a SENDO $(S ;\{\wedge\} \cup E)$ is equationally compact iff for each $f \in E$ the $\operatorname{SENDO}(S ; \wedge, f)$ is. This is to be contrasted with the situation for unary algebras, in which the equational compactness of an algebra with two unary operations cannot be inferred from that of both its mono-unary reducts; such an algebra has been constructed by G. H. Wenzel [8] (and is quoted in [2]).

(4) The terms which are equated in forming the equations under consideration may involve not just the endomorphisms in $E$ individually but also their arbitrary finite compositions; put another way, the same terms would be obtained if $E$ were enlarged to be the submonoid it generates in the endomorphism monoid on which it operates. More generally, one could consider the structure (analogous to that of a module over a ring) of a semilattice on which a monoid operates as endomorphisms. Inspection of the above proof of (ii) $\rightarrow$ (iii) will show that both the embedding and the retraction there constructed preserve the additional structure of those functional compositions with respect to which $E$ is closed.

\section{REFERENCES}

1. S. Bulman-Fleming, On equationally compact semilattices, Algebra Universalis 2 (1972), 146-151.

2. A A note on equationally compact algebras, Algebra Universalis 4 (1974), 41-43.

3. S. Bulman-Fleming and I. Fleischer, One-variable equational compactness in partially distributive semilattices with pseudocomplementation, manuscript.

4. G. Grätzer, Universal algebra, Van Nostrand, Princeton, N.J., 1968.

5. G. Grätzer and H. Lakser, Equationally compact semilattices, Colloq. Math. 20 (1969), 27-30.

6. J. Mycielski, Some compactifications of general algebras, Colloq. Math. 13 (1964), 1-9.

7. W. Taylor, Review of several papers on equational compactness, J. Symbolic Logic 40 (1975), 88-92.

8. G. H. Wenzel, Equational compactness in universal algebras, Habilitationsschrift, Mannheim, 1971.

Department of Mathematics, Wilfrid laurier University, Waterloo, Ontario, Canada

Centre de Recherches Mathématiques, Université de Montréal, Montréal, P.Q., Canada

Technische Hochschule, 61 Darmstadt, Federal Republic of West Germany 